\title{
DOSSIER
}

\section{Musealización de las prácticas artísticas contemporáneas. Colecciones y archivos: nuevas estrategias para su gestión, promoción y difusión}

\author{
Musealization of contemporary artistic practices. Collections and \\ files: new strategies for its management, promotion and dissemination
}

\author{
José Ramón Alcalá Mellado \\ Universidad de Castilla-La Mancha
}

Recibido: diciembre 2016

Aprobado: enero 2017

\section{Resumen}

La sucesión de movimientos y prácticas artísticas desde la segunda mitad del siglo pasado deja un conjunto de creaciones cuyas producciones han abandonado el territorio de lo objetual y lo tangible. A la dificultad intrínseca para su coleccionismo y musealización, se suma la complejidad de estas nuevas propuestas desarrolladas por los artistas durante este periodo, quienes han expandido la idea de arte hasta límites que desbordan los escenarios tradicionales, poniendo en cuestionamiento y modificando de manera sustancial la mayoría de los paradigmas sobre los que se sustentaba. Nos enfrentamos pues a un nuevo e inédito escenario que reclama otro tipo de relación con aquello que tratamos de seguir denominando "obra de arte", exigiendo nuevos análisis, nuevas metodologías para su estudio, difusión y divulgación.

Dentro de esta compleja situación hay que situar la posibilidad de coleccionar estas nuevas producciones. El conjunto de las mismas constituye un valioso patrimonio que, por sí mismo, describe una época -la manera en la que vivíamos, pensábamos e interactuábamos con la realidad-. El museo fue inventado para cumplir esta función: dar acceso público al relato que constituye toda colección artística. Ahora surge la necesidad de repensarlo para que pueda seguir cumpliendo esta importante función.

Palabras Clave: Patrimonio artístico, Coleccionismo artístico, Museografía del arte contemporáneo, Media Art, Arte Contemporáneo.

\begin{abstract}
The sequence of movements and artistic practices since the second half of the last century leaves a set of creations whose productions have left the territory of the objectual and tangible. To the difficulty for being them collected and museographed, it had to be added the complexity of these new proposals developed by the artists during this period, who expanded the idea of art to limits that go beyond the traditional scenarios, questioning and modifying substantially most of the paradigms on which those where based. Therefore we now face a new and unprecedented scenario that calls for another kind of relationship with that we try to keep calling "work of art". This new scenario demands new analysis and new methodologies for the study, dissemination and outreach of all these new art practices.

Within this complex situation must place the possibility of collecting all these new productions. The set of them constitutes a valuable heritage that, by itself, describes a particular time -the specific way we lived, thought and interacted with reality. The museum was invented to fulfill this role: providing public access to the story that inherently contains any art collection. Now arises the need to rethink it so that it can continue to perform this important function.
\end{abstract}

Key Words: Artistic heritage, Art collecting, Contemporary art museography, Media Art, Contemporary Art. 
Este artículo recoge resultados de la investigación de los proyectos de Excelencia I+D "Creación y Estudio de las CAAC (Colecciones y Archivos de Arte Contemporáneo) de Cuenca como modelo metodológico para una investigación de Excelencia en Bellas Artes", financiado a través de la convocatoria del Programa estatal de fomento de la investigación científica y técnica de Excelencia. Subprograma estatal de generación de conocimiento 2014-2016. Ministerio de Economía y Competitividad (MINECO Referencia: HAR2013-48604-C2-1-P); y del Proyecto "Las colecciones de arte electrográfico y digital del MIDE/CAAC. Gestión, conservación, restauración y divulgación de sus fondos", financiado a través de la Convocatoria de Proyectos plurianuales de investigación científica 2014-2017. Fondos europeos FEDER de la Junta de Comunidades de Castilla-La Mancha. (Referencia: POII-2014-002-P), ambos con José Ramón Alcalá Mellado como IP. La traducción al inglés del presente artículo se ha financiado gracias a la convocatoria de Ayudas 2016 a Grupos de Investigación del Vicerrectorado de Investigación de la UCLM.

\section{Introducción, planteamientos e hipótesis de trabajo}

Desearía comenzar con una aseveración que es fruto de mis años de experiencia profesional en este campo: cualquier patrimonio artístico no puede derivar en colección sin un relato que lo sustente. Esta condición es en la actualidad muy patente en relación con las prácticas artísticas contemporáneas de vanguardia, $\mathrm{y}$, entre éstas, de forma especial con aquellas que utilizan $-\mathrm{o}$ giran alrededor de- los medios y dispositivos tecnológicos (denominadas genéricamente en el mundo anglosajón New Media Art). Éstas están especialmente condicionadas por la ausencia de relatos que las den a conocer y las difundan, lo que ha traído como consecuencia (entre otras principales razones) la ausencia de colecciones específicas y, por tanto, su nula presencia en los museos de arte contemporáneo. Es por ello que, en la actualidad, algunas instituciones artísticas están reclamando a los miembros de la comunidad científica internacional (a través de las numerosas convocatorias para la publicación de artículos en revistas científicas, comunicaciones para congresos y jornadas internacionales, etc.) la búsqueda y construcción de lo que en el mundo anglosajón se denomina Media Art Histories.

Los movimientos acaecidos en torno al Media Art - una de las disciplinas más significativas de entre las prácticas artísticas contemporáneas, y paradigma del arte de intangibles - están — seis décadas después de sus primeras manifestaciones - huérfanos de relatos, de literatura artística que mitifique sus prácticas y a sus autores. El problema —o la dificultad - estriba en que el relato se escribe en primera persona (o sobre documentos originales que permitan su construcción). Requiere contexto; acomodo dentro de la Historia del Arte. La clasificación de estas prácticas y su conservación y musealización no podrán ser por tanto establecidas hasta que sus relatos no estén construidos y pertenezcan ya al acervo colectivo, porque ninguno de éstos es neutral; implica siempre un posicionamiento, una voluntad e interés políticos.

Éste es precisamente el importante y necesario reto al que se enfrentan en la actualidad los museos de arte contemporáneo, así como todas aquellas 
instituciones y centros artísticos de producción que, durante el periodo entresiglos, han contemplado el arte de intangibles - la mayoría incluido dentro de las prácticas artísticas mediales y por tanto, incluye obviamente todo el denominado arte tecnológico, aunque no sólo, ya que, por ejemplo, también pertenecerían a esta categoría prácticas y vanguardias artísticas como la performance y el conceptual-, exponiéndolo, produciéndolo, o coleccionándolo como, por ejemplo, Rhizome, ZKM, Ars Linz Electronica, Whitney Museum, MEIAC Badajoz, y también los casos particulares que hemos tomado como referencia y base ejemplar para el desarrollo de este trabajo de investigación: el del MIDE/CAAC de Cuenca y el de la Fundación Juan March y su colección de Arte Sonoro.

Atender y gestionar arte multimedia y arte de intangibles es pues un reto de gran envergadura al que se enfrentan ahora todos aquellos museos e instituciones que han contemplado entre sus colecciones piezas, proyectos y otras producciones contempladas por estas prácticas artísticas contemporáneas. Este reto no es único ni uniforme, pues varía y tiene especificidades propias en función de la tipología de sus diferentes responsables. Así, por una parte están los Comisarios, a quienes cabe atribuir la tarea de la construcción de los inexistentes relatos - microhistorias-, el establecimiento de políticas críticas e historiográficas específicas, y la implementación de nuevas estrategias expositivas y divulgativas; por otra parte están los Conservadores, siempre sometidos a la necesidad de formarse en nuevos (y difícilmente accesibles) conocimientos técnicos e historiográficos, siendo responsables del establecimiento de sólidas políticas de conservación y restauración; y por último, pero no menos importantes dentro del organigrama museográfico, está el Personal de Sala, obligados a realizar un continuo reciclaje profesional, o bien a estar en posesión de una determinada cualificación, necesitando alcanzar una amplia formación técnica y documental, así como la adquisición de unos conocimientos específicos que les permitan adoptar esa deseable actitud diferente que se amolde a las características específicas de estas nuevas prácticas artísticas de vanguardia en el contexto de su exhibición en el museo.

El autor utiliza como trabajo de campo para la elaboración del presente estudio el caso particular de la creación, gestión y divulgación de los fondos patrimoniales incluidos en las Colecciones y Archivos de Arte Contemporáneo (CAAC) de la ciudad castellano-manchega de Cuenca, concebido como un proyecto de renovación de la museografía tradicional desde su creación, en noviembre de 2012, entendiendo que este puede servir como ejemplo de la responsabilidad que conlleva para la institución arte la gestión, preservación y divulgación del patrimonio artístico y cultural que constituye la producción del arte contemporáneo más vanguardista (y por tanto, no asimilado todavía por el mundo del arte) y que se haya podido ir generando en torno a los diversos centros de creación y producción que estas mismas crearon y que han estado gestionando durante las últimas cuatro décadas, durante el periodo de entre siglos. 


\section{Desarrollo}

\subsection{Historificación de las prácticas artísticas de vanguardia. El papel de la institución arte}

La sucesión de movimientos y prácticas artísticas acaecidas desde la segunda mitad del siglo pasado deja un conjunto de creaciones cuyas producciones han abandonado el territorio de lo objetual, lo físico y lo tangible. A la dificultad intrínseca que estas nuevas características suponen para su coleccionismo y musealización, se suma la complejidad de estas nuevas propuestas desarrolladas por los artistas durante este periodo, quienes han expandido la idea de arte hasta límites que desbordan los escenarios tradicionales, poniendo en cuestionamiento y modificando de manera sustancial la mayoría de los paradigmas sobre los que se sustentaba. Nos enfrentamos pues, a un nuevo e inédito escenario que reclama otro tipo de relación con aquello que tratamos de seguir denominando "obra de arte", exigiendo nuevos análisis, nuevas metodologías para su estudio, difusión y divulgación. Dentro de esta compleja situación hay que situar pues la posibilidad de coleccionar estas nuevas producciones. El conjunto de las mismas constituye un valioso patrimonio que, por sí mismo, describe una época, la manera en la que vivíamos, pensábamos e interactuábamos con la realidad.

El museo fue inventado para cumplir esta función y así fue entendido por los grandes pensadores y estadistas: como la mejor herramienta, la mejor arma, para construir una identidad cultural, para grabar en la memoria, en el imaginario colectivo, la personalidad específica de un pueblo, de una nación. El Arte constituye un relato que el museo ha sabido hasta la fecha contar e inocular en el subconsciente colectivo. Ahora surge la necesidad - y por tanto el reto- de repensar el museo - la institución arte-, para que esta pueda seguir cumpliendo su importante función, coleccionando, estudiando, poniendo en valor, gestionando, dando acceso y divulgando el importante patrimonio artístico que han dejado todas estas prácticas artísticas de vanguardia del periodo entre siglos que estamos viviendo y que viene marcada, condicionada, por la transición entre la sociedad y la cultura analógicas hacia las digitales.

Los grandes museos no son aquellos que poseen la mayor cantidad de obras, sino los que pueden presumir de poseer las mejores colecciones, esto es, los que mejor - con mayor inteligencia, astucia, precisión, voluntad y pasión — han sabido construir el Relato - esas micro-historias capaces de conferir valor a las obras individuales, a los movimientos, mitificando a éstas y a sus autores-, a través del cual las colecciones adquieren una personalidad específica, esa autonomía funcional a través de la cual se transmite la Historia, otorgando credibilidad, patente de autenticidad — dando forma en el imaginario colectivo- a sus hitos y leyendas.

De esta forma, la importancia - y por tanto, la necesidad — de coleccionar, 
está ligada a la generación del relato, la construcción de micro-historias. Éstas son importantes porque son el vehículo emocional que conecta la obra de arte con el deseo individual y colectivo. El objetivo de las mismas es que lleguen a afectarnos, que nos emocionen hasta el punto de calar en nuestro inconsciente; ese que nos identifica y distingue culturalmente al convertirse en el notario de nuestra contemporaneidad, como individuos pertenecientes a una época, sociedad y cultura determinadas. La tesis de la película de 2015 Francofonía, del director ruso Alexander Sokurov, explica a la perfección — narrándolo con la precisión del cirujano- la función esencial de los museos en la conformación de nuestra identidad cultural, que legitima la construcción de todo gran imperio, nación.

Desde la segunda mitad del siglo pasado, muchas instituciones públicas y privadas fundaron y abrieron al público centros de producción artística donde poder desarrollar programas actualizados de creación, reflexión y producción en torno a las nuevas prácticas artísticas, y especialmente en torno a aquellas que, por su complejidad técnica, requerían de costosas infraestructuras que dificultaban su acceso de forma individual o también porque sus metodologías creativas reclamaban su colectivización, desbordando la tradicional estructura individualizada. La complejidad propositiva y la inconcreción objetual de muchas de las propuestas que fueron desarrolladas en estos centros generaron un patrimonio artístico que ha quedado bajo el control y explotación exclusivos de estos centros. Con el paso del tiempo, y ante la necesidad de reflejar el panorama creativo de este último medio siglo, los críticos e historiadores del arte han comenzado a reclamar el acceso a estas producciones (la mayoría alternativas a las tradicionales y en su mayoría no exclusivamente objetuales). Pero se han encontrado con que, lamentablemente, muy pocos de estos centros e instituciones tuvieron la precaución, o simplemente la capacidad de comprender, que se habían convertido en depositarias exclusivas de las mismas. Y aquí comienza el problema (que es objeto principal del presente trabajo de investigación). Poseer un valioso patrimonio artístico, todavía no incluido en los estudios historiográficos generales, tal vez por no haber penetrado aún en las colecciones internacionales de arte contemporáneo, exige variadas y complejas tareas museográficas, historiográficas y teórico-críticas a desarrollar en paralelo. Por una parte, es necesario organizar todo este material artístico desconocido, producido probablemente dentro del típico ambiente underground de las vanguardias. Para ello, se debe realizar previamente una descripción de la evolución de las diferentes líneas de acción, reconstruyendo los pormenores domésticos de su historia y de sus creadores, jerarquizándolos en base a su ejemplaridad y significación, y analizando su incidencia en el contexto universal de la construcción de la nueva cultura emergente. Por otra parte, es necesario tratar de conectar el rico mundo proposicional e inédito de estas prácticas artísticas alternativas, no sólo con los estudios de los especialistas y estudiosos, sino también con el público en general (muy importante a la hora de generar un 
nuevo "público", del que surgirá en un futuro, un nuevo "coleccionista").

La implicación de la institución-arte es absolutamente necesaria para este propósito, porque solo a través de esta se puede generar la necesaria normalización de estas prácticas artísticas todavía no asimiladas. La institución-arte (como el museo, el centro de investigación o las colecciones y archivos de obras) tiene la responsabilidad de su preservación en "colecciones", proporciona su acceso al público (especialista y curioso), y trabaja en pro de su proyección histórica. Ésta inicia la conformación de un "patrimonio" (físico y/o virtual), generando las necesarias expectativas de inserción en el mercado oficial del arte. Para los artistas en activo que realizan estas prácticas artísticas, la incorporación de la instituciónarte al escenario general lleva aparejada una serie de ventajas recíprocas: por una parte, el museo/centro de arte provee de las ansiadas audiencias, mientras que, en paralelo, los artistas incorporan a la institución-arte (I.A.) dentro de la cultura más vanguardista. Pero ello exige un cambio de mentalidad por ambas partes: la I.A. debe permitir el uso de sus servidores on-line, libres de censuras, y adaptarse a la nueva y necesaria economía de distribución (enfocada hacia la accesibilidad), mientras que el artista, a través de sus producciones y de su propia personalidad y de la influencia de su "grupo de pensamiento", garantiza audiencias y prestigio, así como la necesaria y deseable credibilidad artística.

Al tratarse de prácticas artísticas de vanguardia tan recientes, generalmente adolecen de la necesaria distancia en el tiempo, esto es, de la perspectiva desde la que generar los análisis teórico-críticos y los estudios historiográficos pertinentes. Es por ello que, en una primera fase, es conveniente la localización, compilación y clasificación de todo el material disponible, así como la transcripción de las historias (micro-relatos) narradas en primera persona; esto es, el acopio de materiales documentales que puedan ir a su vez acompañados de aquellos micro-relatos que los expliquen, den énfasis y seleccionen y distingan entre la totalidad localizada, disponible. Para esta primera tarea, tal vez sean los propios artistas los agentes más apropiados. Su gran sensibilidad, su fino olfato, el conocimiento directo de la cuestión, y la propia experiencia, los convierte en agentes localizadores y discriminadores de este material aún oculto y sin "valor aparente". Además, muy probablemente, estos hayan tenido contacto directo o podido ser co-protagonistas con sus artistas y sus obras en cuestión, lo que sin duda otorga a sus micro-relatos verosimilitud y credibilidad. Estos trabajos hoy día deben ser realizados en los departamentos de investigación de los museos, centros de arte contemporáneo y universidades.

En el caso particular de las prácticas mediales, que han conformado lo que los historiadores del arte han definido y etiquetado de forma genérica como Media Art, estas se han desarrollado desde mediados del siglo pasado, coincidiendo con la aparición y comercialización de las primeras grandes tecnologías audiovisuales, a saber: la cámara de vídeo portátil, la fotocopiadora xerográfica 
y la computadora gráfica. Las producciones que los artistas de las vanguardias más radicales realizaron mediante su uso han tenido una difícil musealización debido principalmente a la ruptura con los parámetros tradicionales que se venían utilizando hasta entonces para su coleccionismo y musealización (exhibición y distribución). No en balde, su irrupción en el escenario de las vanguardias artísticas provocó la aparición de elementos perturbadores de gran magnitud. Los artistas del Media Art comprendieron de inmediato que estas nuevas producciones requerían nuevos sistemas de distribución y exhibición, pero, al no encontrar alternativas viables (como en su día hizo el cine), acabaron por sentirse seducidos, rindiéndose a las ventajas que les ofrecía el museo y la institución arte tradicionales, entre ellas, su glamour, su habilitación social para la sacralización del arte y la inmensa capacidad de difusión en la época de los mass media y de las industrias culturales. Aunque para ello tuvieron que hacer enormes sacrificios, como una perversa adaptación de los objetivos iniciales de sus revolucionarias creaciones y una omisión de muchos de los puntos programáticos de sus Manifiestos. Pero, dado que el museo comprendió rápidamente las ventajas de contemplar y acoger estas nuevas creaciones, de alguna manera incompatibles con sus principios y sistemas funcionales, este también hizo sus sacrificios, con lo que hemos llegado a ese punto intermedio en el que, como ya hemos comentado, ambas partes encuentran sendos beneficios mutuos en esta "relación de conveniencia".

A pesar de este encuentro, a pesar de este convencimiento mutuo de contemplarse y respetarse, el panorama no es demasiado alentador. Todavía hoy no existen prácticamente obras de Media Art expuestas en los grandes museos de arte contemporáneo del mundo, porque apenas están representadas en sus colecciones, y si lo están, estas no suelen ser exhibidas porque, por una parte tienen un difícil y complejo montaje y mantenimiento y, por otra, y tal vez la razón más importante y decisiva, porque todavía no han encontrado acomodo en el relato general de la Historia del Arte Contemporáneo al no haberse generado los necesarios micro-relatos que provean a estas obras de una literatura artística que mitifique a sus artistas y a sus producciones, creando ese necesario interés y deseo hacia ellas. Además, en la actualidad solo se mantienen y siguen funcionando en todo el mundo media docena de centros que se dedican en exclusiva a la producción y apoyo al Media Art. Los Medialabs, herederos de los Centros de Arte y Nuevas Tecnologías de la segunda mitad del siglo pasado y que prendieron con fuerza en las grandes metrópolis con la entrada del nuevo siglo, han dejado de ser centros de investigación y producción de Media Art, para convertirse en laboratorios ciudadanos de mediación cultural (en los cuales la creación artística es sólo un aspecto tangencial, por no decir residual, del conjunto de sus objetivos y actuaciones). Es decir, centros donde la utilización de los medios y dispositivos tecnológicos - hoy digitales o electrónicos - está puesta al servicio de las necesidades y retos sociales en general, o, en el supuesto más favorable tal y 
como algunos responsables defienden dentro de estos medialabs actuales, de un arte que ha expandido tanto sus límites y campos de actuación que se ha fundido transdisciplinarmente con el resto de las disciplinas científicas.

La aparición de Internet y la conformación del Net.Art como movimiento artístico a comienzos de los 90 cambiaron la mayoría de los parámetros del escenario de las prácticas artísticas, dejando como resultado un patrimonio artístico totalmente inédito y alternativo al tradicional contemplado en las colecciones y museos de arte contemporáneo. Sus nuevas reglas de juego propuestas por los net-artistas la virtualización y deslocalización de sus producciones han creado una enorme convulsión en el mundo del arte, despistando a sus gestores y alejando de su ámbito de interés a críticos e historiadores.

A la evidente dificultad de asumir el patrimonio artístico de lo intangible que ha sido o está siendo producido por las muy diversas manifestaciones de los Media Art, sobre todo si se intenta aplicar las mismas reglas de funcionamiento y parámetros de actuación que con las tradicionales objetuales, se suma la lentitud de la que siempre ha hecho gala la institución arte, que además tropieza —una vez más en la Historia del Arte - con la complejidad formal de sus planteamientos. Incluso cuando el Media Art se formaliza a través de instalaciones objetuales (o de naturaleza híbrida) coincidiendo con la ambición máxima del Museo, el objetualismo, sus montajes plantean una enorme complejidad, requiriendo espacios e infraestructuras que el museo no suele poseer; a lo que hay que añadir la falta de equipamientos adecuados y de un personal técnico y de mantenimiento altamente especializados. Por otra parte, y no menos importante, son las constantes colisiones éticas y morales que se producen entre los planteamientos y actitudes de los mediaartistas (sobre todo los net-artistas) y los principios que rigen la institución-arte. Además, hay que contar también con la arenga que constantemente realizan las nuevas - y alternativas - Comunidades-Red contra las viejas Instituciones-Arte.

\subsection{Coleccionar lo inmaterial. La necesidad de construir nuevas estrategias museográficas para la gestión y divulgación del patrimonio originado por las prácticas artísticas de vanguardia de entre siglos}

En este difícil y complejo contexto hemos de situar la intención por la gestión y preservación del patrimonio artístico, no solo de lo intangible, sino de muchas de las producciones de las nuevas prácticas artísticas de vanguardia, entre las que destacan de manera especial (aunque solo sea por su cantidad) aquellas creaciones que utilizan los dispositivos tecnológicos y que se agrupan bajo la denominación genérica internacional de Media Art.

Tal y como hemos justificado, los museos y centros de arte contemporáneo deben asumir la responsabilidad institucional de su preservación, promoción y difusión, lo que implica repensar su función y su funcionamiento. Para ello, por una parte está la difícil tarea de asumir una política de coleccionismo consecuente, 
que lleva aparejada una serie de fases que hay que concebir y ejecutar con la necesaria coherencia. Por una parte, los responsables del museo o de su patronato deben de embarcarse en un proceso de selección. Esto es, seleccionar piezas significativas (ejemplares), cuestionándose en cada caso, si esto es Arte y si la pieza en cuestión es en potencia históricamente significativa. Por otra parte, hay que redefinir el papel del comisario, como filtro y como facilitador), y asumir que en este proceso la institución-arte va a actuar —en términos históricoscomo legitimadora de todas estas obras, piezas y producciones (apelando a su incuestionable prestigio sociocultural). En una fase posterior, la institución-arte se debe enfrentar a las problemáticas inherentes a todo proceso de adquisición (propiedad) de estas obras y piezas seleccionadas. El escenario sigue siendo el mismo, la invariabilidad del deseo de transacción de la propiedad. Pero, en el caso particular del arte de intangibles, surgen nuevas problemáticas (o, al menos, se introducen nuevas variables hasta entonces nunca antes contempladas), como son los derechos adquiridos para la recirculación de la información, la todavía ineficiente legislación de los derechos de autor propiedad intelectual y reproducción, así como la creación de entornos de preservación que aseguren su accesibilidad, su conservación y mantenimiento permanente (luchando contra la obsolescencia propia de los dispositivos tecnológicos). Contra estas se oponen, por ejemplo, el constante empeño de los artistas por alterar sistemáticamente las condiciones de autoría, de producto y de flujo de la información, la imposibilidad de adecuar la nueva situación y condiciones a las prácticas mercantilistas tradicionales, o la necesidad de implementar y gestionar un nuevo concepto de Patrimonio Artístico pues, en la mayoría de los casos, más que adquirir, la institución-arte deberá aspirar a conseguir los derechos de circulación de estas nuevas producciones, dentro de una nueva economía que no esté sustentada en la concepción material de la obra como mercancía sino en el acceso, y por tanto en las estrategias y políticas de su distribución y difusión. Otro escenario que queda sustancialmente modificado a la hora de patrimonializar estas nuevas prácticas y producciones artísticas es el de la organización (que atiende a su clasificación y conservación). Surgen ahora nuevas problemáticas y retos, como son, por ejemplo, la dificultad de dibujar una cierta taxonomía posible (ausencia de un tesauro específico), que tropieza con la enorme diversidad de propuestas, objetivos y actitudes, o la dificultad de ser usuario de estas piezas y obras de arte debido, principalmente, a la diversidad de diseños de sus interfaces y sistemas de navegación y de sus múltiples niveles de interactuación. A lo que hay que sumar esa aspiración por parte de sus creadores por escapar a los modelos discursivos normalizados (tratando de seguir y explotar hasta sus límites la máxima introducida por las vanguardias artísticas de la segunda mitad del siglo XX de ARTE = VIDA). Existe además una ausencia significativa de estudios críticos y metodológicos, tal vez por la dificultad de acceso directo a estas creaciones. ¿Clasificar? Incluso el propio concepto ha sido en más de una 
ocasión puesto en cuestionamiento para este tipo de nuevas prácticas artísticas por parte de algunos de los escasísimos críticos de arte especializados. En este sentido, el filósofo español, crítico y comisario de Net.Art, José Luis Brea, aseveraba que "la red no es un espacio de archivo, sino de actuación. [...] no tiene sentido producir en ella para la memoria y el rescate [...]. Sino para la intercomunicación, la intertextualidad, los efectos de proceso y comunicatividad" (Brea, 2003, p. 58). En este tremendo lío nos hallamos inmersos en la actualidad. Por último, queda por abordar el siempre complejo y cambiante campo de la divulgación. Promocionar este arte para darlo a conocer, aspirando a que pueda llegar a ser comprensible, a que sus obras y artistas puedan llegar a ser amadas y por tanto deseadas, para que sean de fácil acceso. Aspiraciones que solo podrán ser alcanzadas si se logra una actuación en términos de rentabilidad. Lo que exige que todas estas prácticas artísticas alternativas sean capaces de ser incorporadas dentro de una corriente generalizada, universal y fuera de las "modas". Para ello, la institución arte debe trabajar en pos de la consecución de un Estado de Opinión para atraer audiencias, de la conformación de una nueva e inédita concepción, creación y diseño de una futurible "galería-museo-laboratorio" capaz de albergar y gestionar todas estas prácticas representativas de las vanguardias de entre siglos (virtuales o no). En cualquier caso, entre los retos y dificultades que ahora se presentan para una adecuada difusión de todo este patrimonio de arte intangible, figuran la construcción de una adecuada legislación que proteja todas estas actuaciones, teniendo en cuenta que la ley de los nuevos medios, es la ley del "tiempo real", en donde esté incorporado y asumido el concepto de "presencia" como garante del "tiempo del acontecimiento". Ya no se trata de producir los dispositivos necesarios para lograr emitir dichas producciones, sino de hacerlas visibles. En palabras de José Luis Brea: "En la sociedad del siglo 21 no será ni necesario —ni casi posible - coleccionar obras de arte (como ya es espurio coleccionar cine o música) y la función de las instituciones públicas respecto a las nuevas prácticas, con vistas a garantizar su inscripción en lo público, será más bien la de promover u optimizar en entornos protegidos la circulación social de aquellos contenidos que el libre mercado de las industrias culturales desestimaría en su regulación interesada por la ley de la audiencia" (Brea, 2003, p. 123).

En la década de los noventa surgieron en los países más desarrollados los primeros centros artísticos orientados exclusivamente hacia la producción de propuestas lideradas por artistas que utilizaban o reflexionaban en torno a los nuevos medios y dispositivos tecnológicos. Tan solo una década después, la mayoría de estos cerraron sus puertas y solo unos pocos mantuvieron su actividad al comenzar el nuevo siglo. Entre estos, centros como el ZKM en Karlsruhe, Ars Electronica Center en Linz, la Fondation Daniel Langlois en Montreal o el Museo Internacional de Electrografía - Centro de Innovación en Arte y Nuevas Tecnologías (MIDECIANT) en Cuenca, además de seguir apoyando la producción de New 
Media Art, tomaron conciencia de su responsabilidad institucional como gestores del inmenso patrimonio de Media Art que habían ido atesorando en sus talleres, aulas, departamentos y laboratorios. Las obras, producciones y documentación que conforman las colecciones que gestionan poseen unas características específicas que las diferencian del resto de colecciones de arte que han ido creando los museos de arte contemporáneo. Entre estas cabe destacar la virtualidad (o con mayor precisión, la no-objetualidad o inmaterialidad) de la naturaleza de muchas de sus creaciones y piezas de sus colecciones y archivos, particularmente las propiciadas por el uso de las nuevas tecnologías eléctricas y posteriormente digitales y electrónicas. Videoarte, infografía, o las más variadas manifestaciones del arte electrónico son algunas de sus disciplinas más representativas. Pero también es de naturaleza inmaterial cuanto fue —o es todavía hoy— producido por las prácticas accionistas y performativas, por la manipulación del sonido como materia prima artística, así como por las que devienen de la mayoría de los proyectos desarrollados dentro del arte conceptual. Asimismo, y aunque su aportación no fue tan radical como la de las que acabamos de mencionar, ya que, a diferencia de aquellas, la naturaleza de sus producciones no era inmaterial sino objetual, hay que incluir entre estas nuevas prácticas de difícil musealización aquellas en las que los artistas tomaron en consideración o hicieron uso para sus producciones de las tecnologías electrográficas y los procesos de multi-reproducción de imágenes, y que dieron lugar en esa misma época a movimientos internacionales de gran actividad e influencia, como es el caso especialmente significativo y relevante del copy art y el fax art. Estos tomaron prestadas las aportaciones conceptuales que había establecido - lentamente y con enorme dificultad - una fotografía ya madura, la cual, tras más de 150 años de existencia, había conseguido por fin ser aceptada como técnica y lenguaje artísticos, introduciendo nuevos parámetros que colaboraron decisivamente en el desmantelamiento - o al menos, en la puesta en cuestionamiento- de algunos de los principales paradigmas de las prácticas artísticas tradicionales (la del arte moderno occidental, hegemónico desde el siglo XIV); a saber: la dialéctica original-copia, el concepto de autoría y el de espectador, o el fin de la primacía de la imagen, entre los más significativos.

El resultado de todos estos nuevos planteamientos creativos fue la aparición y desarrollo de toda una serie de prácticas artísticas experimentales que se infiltraron entre las vanguardias "oficiales" del arte de entre siglos, aportando parámetros inéditos a los discursos y lenguajes tradicionales, y generando un escenario lleno de fracturas y disonancias, al estar estructuradas sobre nuevos paradigmas que desmantelaban los tradicionales sobre los que se había erigido el mundo del arte durante los anteriores seis siglos. Todo resultaba inédito y estaba necesitado de nuevos y alternativos ecosistemas: sus ideas, sus prácticas y sus lenguajes y disciplinas, que iban a propiciar como consecuencia de su aplicación en los procesos creativos otro perfil de artista y de público, y por tanto, otros 
sistemas de difusión, valoración y distribución: videoarte, prácticas accionistas y performativas, arte sonoro, electrografías artísticas, arte digital y electrónico, y - como movimiento específico de estos últimos-Net.art.

Junto a los mencionados centros de "arte tecnológico" que, en el periodo de entre siglos, tomaron conciencia de su responsabilidad como coleccionistas de estas nuevas prácticas experimentales, aparecen algunos centros universitarios. Muchas de estas creaciones experimentales se generaron, desde la década de los años cincuenta del siglo XX, en torno a los departamentos de arte y diseño de las más avanzadas universidades occidentales, dentro de un contexto elitista $\mathrm{y}$ radicalmente vanguardista propiciado por los nuevos centros de creación artística financiados por instituciones públicas y privadas y corporaciones multinacionales (casi todas ellas dedicadas a la fabricación y comercialización de productos tecnológicos).

\subsection{El modelo museográfico de las Colecciones y Archivos de Arte Contemporáneo (CAAC) de Cuenca}

Uno de sus ejemplos más relevantes dentro del panorama internacional fue el de la Facultad de Bellas Artes de Cuenca. La fundación, a mediados de los años ochenta del siglo pasado, de una nueva facultad de bellas artes por parte del gobierno de la joven comunidad de Castilla-La Mancha en una zona periférica rural del centro del estado español contó con un Plan de Estudios altamente experimental, que se quiso vincular inicialmente a las ideas renovadoras de los artistas del arte abstracto español, muchos de ellos afincados desde dos décadas antes en dicha ciudad. Para ello, y con el fin de dar continuidad a las ideas fundacionales del revolucionario Museo de Arte Abstracto Español de Cuenca, el gobierno regional, a través del equipo rectoral encargado de la creación de la Universidad de Castilla-La Mancha (UCLM), nombró una comisión compuesta por algunos de estos artistas abstractos ligados a Cuenca (entre los que se encontraban, entre otros, Gustavo Torner, Lucio Muñoz, Julio López Hernández, Luis Gordillo, Rafael Canogar o José María Yturralde), encargándoles su ideación y puesta en funcionamiento. Estos tomaron la acertada decisión de contratar como profesores a algunos de los más jóvenes artistas, críticos e historiadores del panorama español de la época que eran especialistas en disciplinas, lenguajes y movimientos artísticos más vanguardistas y alternativos del momento. Nada más tomar posesión de sus cargos docentes, estos pusieron en marcha una serie de incitativas y actividades presididas por una gran pasión y activismo, llegando a fundar dentro de la propia Facultad (o fuera de esta, como es el caso del MIDE), talleres y laboratorios experimentales donde se desarrollarían proyectos alrededor de todas estas nuevas prácticas artísticas alternativas a las vanguardias "oficiales".

Desde su inauguración, en el curso 1986-87, la Facultad de Bellas Artes de Cuenca pondría especial atención al desarrollo de todas estas nuevas prácticas y 
lenguajes, creando un entorno de experimentalidad, tan radical como alternativo, que contagió al resto de facultades, escuelas y departamentos artísticos del país. Pero la ventaja de esta nueva facultad frente a las demás era precisamente su insultante juventud. Con un claustro de profesores que apenas superaba los treinta años de edad media y sin embargo todos ellos ya reconocidos artistas y activos militantes dentro de algunas de estas disciplinas mencionadas, el ambiente vanguardista y alternativo que se respiraba en sus pasillos, aulas y talleres era imposible de empatar por parte de la mayoría de las "grandes" facultades artísticas españolas, lo que generó un éxodo de alumnos desde aquellas hacia la de Cuenca, buscando ese "aire fresco" que, en esa época, no podía ser respirado en Barcelona, Madrid o Sevilla. Con una filosofía de permanentes puertas abiertas, programas de actividades complementarias que atraerían la presencia de los artistas, profesores y alumnos más vanguardista de la época, inoculando una actividad altamente creativa, inspiradora y contagiosa, la Facultad de Bellas Artes Cuenca puso en funcionamiento talleres y centros experimentales y hasta un museo abierto al público de forma permanente y con sede en uno de los edificios históricos más emblemáticos de la ciudad. Así se crearon, desarrollando una frenética actividad artística paralela a los museos y centros de arte contemporáneo al uso, el Museo Internacional de Electrografía de Cuenca (MIDE) (1989), el Centro de Creación Experimental (1990), el Instituto de Estudios Avanzados de Comunicación Audiovisual (1996). Se pusieron en marcha recursos de acceso público, libre y gratuitos como la radio experimental Radio Fontana Mix (1993), el repositorio de Net.art Aleph-Arts.org (1996), IDECA (Investigación y Desarrollo de Contenidos Audiovisuales) (1999) o el Archivo Virtual de Artes Escénicas (dentro del proyecto ARTEA) (2003). Y se organizaron eventos públicos periódicos, como el congreso "La Situación" (1993) o, desde el año siguiente a su celebración, el festival Situaciones. Un enorme y variado conjunto de recursos para la experimentación práctica y la investigación teórico-crítica funcionando a pleno rendimiento durante toda la década de los noventa, época en la que, para el resto de las instituciones arte (sobre todo las académicas), la práctica artística aún quedaba limitada a las disciplinas y prácticas tradicionales de la pintura, la escultura, el grabado y a las entonces novedosas y polémicas del diseño y la publicidad.

Tras más de dos décadas de intensa actividad, la Facultad de Bellas Artes de Cuenca se encontró, a finales de la primera década del presente siglo, inmersa en una profunda crisis de identidad, al haber alcanzado su mayoría de edad, potenciada por la brutal parálisis económica nacional, que le obligó a clausurar la mayoría de sus talleres laboratorios, recursos y actividades experimentales. Tras un profundo análisis colectivo y haciendo un saludable ejercicio de autocrítica, el claustro del centro decidió por unanimidad, en noviembre de 2012, poner en funcionamiento un nuevo y revolucionario proyecto: la creación y desarrollo de las Colecciones y Archivos de Arte Contemporáneo (CAAC) de Cuenca (www.caac-uclm.es). 
Para entonces, la Facultad contaba además con una serie de nuevas colecciones, fruto de donaciones externas fundamentadas, en la confianza que este centro se había granjeado entre los actores principales del mundo del arte contemporáneo. Por ejemplo, la prestigiosa galerista y coleccionista de arte contemporáneo Helga de Alvear, donó en 2010 a la Facultad de Bellas Artes de Cuenca dos importantes colecciones, las cuales habían sido previamente entregadas al Museo Nacional Reina Sofía (MNCARS) y que, visto el escaso rendimiento y la nula atención que se les estaba dando, optó por retirarlas del museo nacional y legarlas al centro universitario, bajo la promesa de que en esta estarían permanente expuestas, convirtiéndose en recursos vivos para la enseñanza artística y organizándose anualmente exposiciones y muestras en torno a sus valiosos fondos. De esta forma, la Colección de Arte Múltiple devenida de la edición de la revista internacional de arte contemporáneo Parkett (editada en Zúrich y con delegación también en Nueva York) pasaba a sumarse a los fondos de las colecciones, archivos, documentación y recursos devenidos de todas las actividades desarrolladas en los centros, laboratorios y actividades de la Facultad de Cuenca descritas. A la Colección Parkett se sumó también la Colección de Grabado Contemporáneo que atesoró la Galería Juana Mordó durante todos sus años de actividad y que había sido heredada por Helga de Alvear de su primera dueña (al haber sido la responsable de la galería madrileña durante su última etapa), y que ahora donaba también a la Facultad de Cuenca.

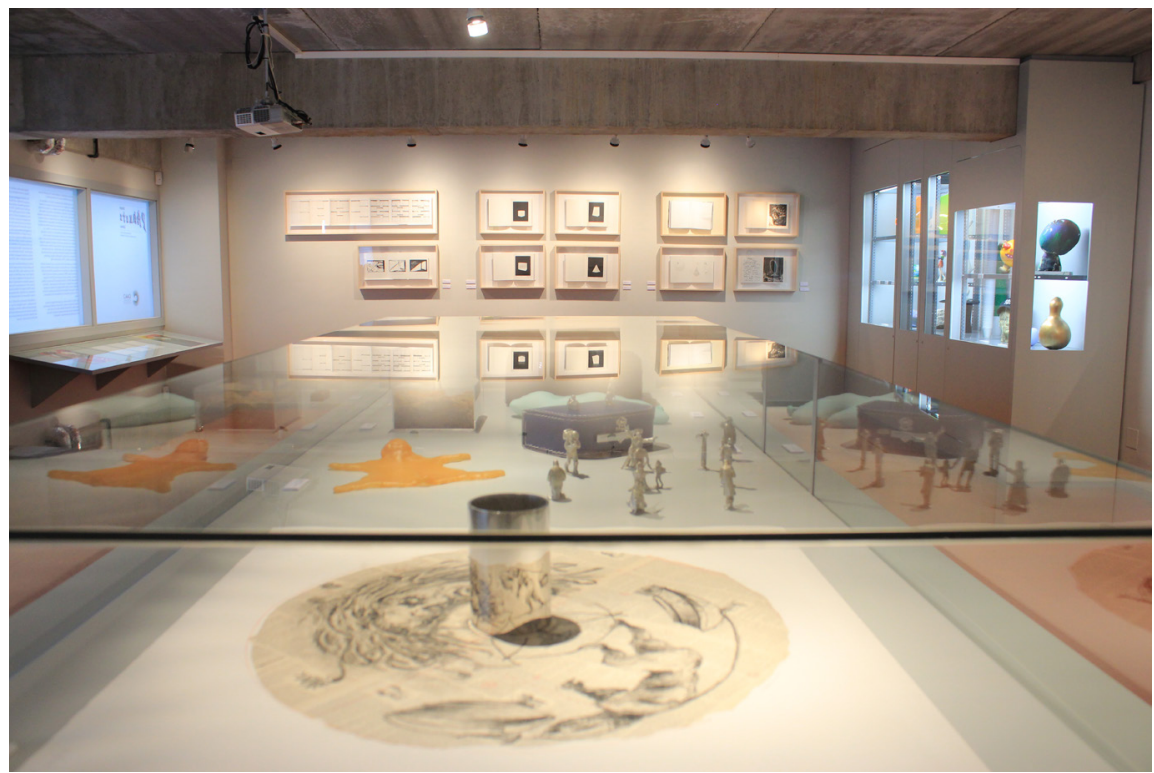

Imagen 1. Sala Parkett. Exposición Nuevos planteamientos de investigación en las CAAC. Pasado, presente y futuro comisariada por Beatriz Escribano y Cristina Peña. En la $1^{\text {a Jor- }}$ nada de las CAAC, Facultad de Bellas Artes de Cuenca, 26 de abril de 2016. Imagen cedida por las CAAC de Cuenca. 
Una vez creado el proyecto de las CAAC_Cuenca, sus responsables localizaron e incorporaron los Archivos Cinematográficos de la productora El Deseo S.A., donados a la UCLM por el cineasta manchego universal Pedro Almodóvar y su hermano Agustín - su productor-, como agradecimiento por su nombramiento como Doctor Honoris Causa de la UCLM a propuesta del Departamento de Arte de la Facultad de Bellas Artes de Cuenca. Asimismo, y con el propósito de ampliar el contexto específicamente universitario de los archivos y colecciones de las CAAC_Cuenca, conectándolo con su ámbito natural geo-histórico, y que hace a la Facultad de Bellas Artes heredera de aquel espíritu vanguardista inaugurado en Cuenca por los artistas del grupo El Paso a través de la creación del visionario Museo del Arte Abstracto Español de Cuenca (creado veinte años antes que aquella), se incorporaron otras colecciones creadas fuera de la UCLM. Este es el caso del histórico Gabinete de Música Electroacústica (GME) de Cuenca, puesto en marcha y financiado por su Diputación Provincial a comienzos de la década de los noventa, ubicado inicialmente en el Conservatorio de Música de la ciudad. Después de una compleja labor llevada a cabo a comienzos del presente siglo XXI por las asociaciones AVADI y ACUOSO, y, desde 2014, por "Acción GME v2.4", y culminadas por el grupo de investigación del Departamento de Arte de la UCLM "FUZZY GAB .4", se consiguieron localizar los fondos, infraestructuras y equipos pertenecientes al GME. Tras más de dos años de complejas gestiones con sus propietarios legales, los responsables de las CAAC_Cuenca, consiguiendo la confianza de éstos, lográndose la firma de un Convenio de Colaboración entre los responsables académicos de la UCLM y la Presidencia de la Diputación de Cuenca mediante el que ésta traspasaba la gestión, promoción y divulgación del mismo a las CAAC Cuenca, y ubicando el GME en los laboratorios de Arte Sonoro de la Facultad de Bellas Artes. El famoso sintetizador Synthi 100 (casi único en el mundo; sólo se conserva en la actualidad uno más, localizado en Francia), y toda la música electroacústica creada a lo largo de la década de los noventa a través de éste por los más prestigiosos músicos internacionales (como, por ejemplo, Luis de Pablo, César Cano, José Iges, Concha Jerez, José Manuel Berenguer, Eduardo Polonio, o Gabriel Brnçic, quien había sido su director, entre otros) eran por fin rescatados del ostracismo y del más absoluto abandono, incorporándose también a los fondos, colecciones y recursos de las CAAC_Cuenca, a finales del curso 2016-17 y pasando a ser gestionados desde entonces por el grupo FUZZY GAB .4, gracias a la financiación propiciada por los proyectos de investigación de Excelencia MINECO (HAR2013-48604-C2-1-P) y el plurianual de la Junta de CLM con fondos europeos FEEDER (POII-2014-002-P).

Las CAAC_Cuenca suponen pues el esfuerzo y el empeño individual y colectivo de una serie de artistas-activistas profesores-investigadores de la Facultad de Bellas Artes de Cuenca por asumir, desde y en representación de la institución arte, la responsabilidad de gestionar ese nuevo Museo de Artistas 
(curiosamente inaugurado históricamente en el panorama internacional por el Museo de Arte Abstracto Español de Cuenca, hace ahora exactamente medio siglo) (1). El objetivo es por tanto tratar de relacionar todas estas prácticas artísticas, así como sus experimentaciones e investigaciones, con la museografía del arte contemporáneo, asumiendo, gestionando, promocionando y divulgando estas para que puedan integrarse en el relato general de la Historia del Arte, de tal manera que posibilite la conformación de una serie de colecciones que puedan integrarse de manera normalizada en el museo, aunque para ello la institución arte deba haber hecho "sus deberes", esto es, actualizarse y reinventarse. Esto es lo que el Museo Internacional de Electrografía de la UCLM en Cuenca, en particular, $\mathrm{y}$, en general, todas las demás colecciones, archivos y recursos que conforman en la actualidad las CAAC de Cuenca como micro-museos de artistas del siglo XXI, están planteando y desarrollando. Para estos, su consolidación, proyección y repercusión pasa necesariamente por la capacidad que se tenga de construir el relato (las micro-historias particulares) que conecte la Historia de la Facultad de Bellas Artes y de sus diferentes colecciones y archivos generados en sus propios laboratorios y talleres (sumados a las heredadas provenientes de donaciones particulares) con la historia particular de los movimientos de las diferentes vanguardias artísticas de la segunda mitad del siglo XX que tomaron protagonismo y se vincularon a la ciudad de Cuenca y su entorno geográfico y cultural, para conectarlas de forma definitiva con el relato general "oficial" de la Historia del Arte Contemporáneo y sus diferentes movimientos de vanguardia.

$\mathrm{Su}$ arriesgada e innovadora propuesta se presenta como un nuevo modelo alternativo a las estrategias museográficas del arte contemporáneo capaz de poner en valor, gestionar y divulgar el prolífico - y sin embargo desconocidopatrimonio artístico propiciado por algunas de las más radicales y alternativas vanguardias artísticas que han acontecido (y lo siguen haciendo) durante el periodo que transita entre el siglo XX y el XXI y que comienza con el inicio de la segunda mitad del siglo pasado, en la década de los años cincuenta.

\section{Conclusiones}

Las prácticas artísticas de las vanguardias más radicales del periodo entre siglos han dejado un sinfín de creaciones de difícil musealización, fundamentalmente por atender a nuevos e inéditos parámetros respecto a los tradicionales a los que hasta ahora se han tenido que enfrentar los museos de arte contemporáneo y porque apenas existen relatos al respecto, esas micro-historias de las que se alimenta la literatura artística que promociona los distintos estilos y vanguardias que nutren a las colecciones de Arte.

Pocos son, en la actualidad, los museos-centros de arte que han atendido a esta necesidad y que han tomado la responsabilidad institucional de coleccionar, 
gestionar, conservar, promocionar, dar acceso y divulgar estas creaciones experimentales, tan difíciles de encontrar en los museos de arte contemporáneos actuales.

Entre estos, está el proyecto de las Colecciones y Archivos de Arte Contemporáneo que gestiona la Facultad de Bellas Artes de Cuenca, perteneciente a la Universidad de Castilla-La Mancha. Desde que fuese aprobado por su Junta de Centro, en noviembre de 2012, las CAAC_Cuenca han procurado asumir este reto a partir de su propio patrimonio. Una riquísima y variada colección de obras, creaciones, archivos y documentos de naturaleza heterogénea que fueron producidos en los propios talleres y laboratorios que algunos de los profesores, artistas e investigadores contratados desde 1986 para crear esta nueva $-\mathrm{y}$ revolucionaria - Facultad de Bellas Artes española, realizaron con pasión y militante convicción ejemplares.

El modelo museográfico que proponen las CAAC_Cuenca, a caballo entre un medialab de producción y experimentación, un museo sin paredes que afronta los nuevos retos de acoger las nuevas prácticas artísticas y un centro de enseñanza experimental que funciona como un ágora permanente de intercambio de ideas creativas dentro de una estructura mixta horizontal-vertical, permite aportar ideas innovadoras y sumarse así a las pocas iniciativas que van surgiendo en la actualidad de manera tan tímida como espaciada en los países desarrollados del planeta en torno a sus problemáticas principales, como son, entre otras, la gestión de los derechos de autor y propiedad intelectual, la gestión y conservación de esta complejas piezas, la clasificación, taxonomía y tesauro de sus creaciones (sobre todo en los que se refiere al Media Art), las posibles estrategias expositivas, o la necesidad de implementar nuevas políticas de divulgación y, en general, museográficas.

\section{Notas}

(1) Este término lo emplea por primera vez Manuel Fontán, director de Exposiciones de la Fundación Juan March, para referirse precisamente a una de las especificidades del Museo del Arte Abstracto Español de Cuenca, explicando que este concepto es una invención del museo conquense, creado y concedido por el artista (rico descendiente de terratenientes filipinos) Fernando Zóbel, en 1966.

\section{Referencias Bibliográficas}

Alcalá, J. R. y Jarque, V. (2016). CAAC Cuenca; Centro de Arte, Archivos y Colecciones. Cuenca: Cuenca, la ciudad y la cultura. Cuenca: Ediciones de la Universidad de Castilla-La Mancha. Colección Caleidoscopio, \#12.

Alcalá, J. R.; Fernández, L. y Rico, J.C. (2009). ¿Cómo se cuelga un cuadro virtual? Las exposiciones en la era digital. Gijón: Trea.

Brea, J. L. (2003). El tercer umbral. Estatuto de las prácticas artísticas en la era del capitalismo cultura. Murcia: CENDEAC. 


\section{Cita Recomendada}

Alcalá, J. R. (2017). Musealización de las prácticas artísticas contemporáneas.

Colecciones y archivos: nuevas estrategias para su gestión, promoción y difusión. Sin Objeto, 00, 40-58.

Doi: http://dx.doi.org/10.18239/sinobj_2017.00.03 


\section{Biografía}

\section{José Ramón Alcalá Mellado}

Universidad de Castilla-La Mancha

joser.alcala@uclm.es

José Ramón Alcalá (Valencia, 1960) es catedrático de Procedimientos Gráficos de Expresión y Tecnologías de la Imagen en la Universidad de Castilla-La Mancha. Director del Museo Internacional de Electrografía (MIDECIANT) de Cuenca, desde su creación (1989). Responsable de las "Colecciones y Archivos de Arte Contemporáneo" (CAAC) de la Facultad de Bellas Artes de Cuenca. Responsable del grupo de investigación "Interfaces Culturales; Arte y Nuevos Medios" de la UCLM. Premio Nacional de la Calcografia Nacional (al MIDE) "por las innovaciones aportadas al arte gráfico" (Madrid, 1999). Es Investigador Principal de proyectos I+D+i de Excelencia nacionales y europeos sobre aplicaciones de las nuevas tecnologías en la creación artística y la museografía virtual. Autor de libros, como: La piel de la Imagen (Valencia, 2011); Ser Digital; Manual para conversos a la cultura electrónica (Santiago de Chile, 2011); ¿Cómo se cuelga un cuadro Virtual? (Gijón, 2009); Monstruos, fantasmas y alienígenas. Poéticas de la representación en la cibersociedad (Madrid, 2004); o Ars \& Machina. Electrografía Artística en la colección MIDE (Santander, 2004). Director y comisario de bienales y premios, como el Observatorio Internacional de Artes Electrónicas de Gijón (OOH) http:// www.jornadasooh.net, Digital Art Awards LÚMEN_EX (Universidad de Extremadura) http://www.lumenex.net, Festival de Artes Electrónicas de Valencia Digital Media $1.0 \mathrm{http} / /$ www.digitalmediavalencia.es. Como artista creó, en 1983, el equipo Alcalacanales (con Fernando Ñ. Canales), cuyas exposiciones y actividades relacionadas con la creación artística con nuevos medios se prolongaron en el circuito internacional hasta 1994. 